Application), the remobilization of professionals in this domain within the framework of technical co-operation projects with the IAEA and the AFRA Programme, and the creation on 31 October 2002 of the National Agency for Radiation Protection. This stresses the importance that the Cameroon Government attaches to peaceful applications of nuclear energy in the priority sectors of development in order to fight poverty. The work in progress or in preparation at CEPAMOQ is on radiotherapy (quality control of conformational treatment and IMR, dosimetric calculations using the Monte-Carlo method around radioactive grains), radiology (evaluation of doses of $\mathrm{X}$-rays delivered by helicoidal scanners), nuclear instrumentation and spectrometry (mounting of a gamma detection set up and measuring of radioactivity of environmental samples) and the management of radioactive waste.

\section{Atoms and molecules}

Six lecturers-researchers and six doctoral students are involved in this area. Research of a theoretical nature is oriented towards fundamental studies of atomic physics (life times of heavy radioactive ions including highly ionised lanthanides, atoms in intense laser fields, electron-atom scattering), molecular physics (dissociative recombination of molecular ions, molecules in intense laser field), cluster physics (stability of highly charged metallic clusters).

The interest triggered by CEPAMOQ is demonstrated today by the number of cooperations which have been initiated, and fruitfully implemented. At the national level, CEPAMOQ constitutes the research focal point in nuclear, atomic and molecular physics for lecturers from the five state universities of Cameroon with a scientific nature. Contacts have been established with national Institutions capable of benefiting from our activities: the General Hospitals of Yaounde and Douala, the Institute of Geological and Mining Research (IRGM), the National Committee on Climate Change, the Permanent Secretariat of Environment (Ministry of Environment and Forests), the National Programme for Agricultural Research and Popularisation, the Department of Civil Protection (Ministry of Territorial Administration) and the Mount Cameroon Observatory based at the IRGM.

At the African level, CEPAMOQ already welcomes foreign students and collaboration has started with the Physics Department of the Marien Ngouabi University (Brazzaville, Congo), the Lasers Atoms Laboratory of the Cheikh Anta Diop University (Dakar, Senegal), the Atomic Molecular Spectroscopy and Applications Laboratory of the University of Tunis-El Manar (Tunisia), and the Medical University of Southern Africa (South Africa).

Out of the African continent, CEPAMOQ has singled out itself through research and training missions in European and Northamerican universities and institutions (e.g. Université Paris-Sud, CEA-Saclay, Université de la Méditérranée in France, Université Catholique de Louvain in Belgium, Division of Applied Physics of the ENEA Centre at Frascati and the Abdus Salam ICTP centre in Italy, and the Université de Laval in Canada). Support has been provided by the partner institutions, their Embassies, ICTP and the AUF (Agence Universitaire Francophone).

Chosen to host the $7^{\text {th }}$ LAM (African Laser Atomic Molecular and Optical Science Network) conference, CEPAMOQ hopes to establish its position within the network by succeeding in the organisation of this event planed for December 2004 at the University of Douala.

All these achievements give us hope and and make us ambitious, given the interest we have aroused both from local and international partners. Hence the necessity of supporting this dynamic trend in its success.
"Unless it has its own scientists and

technicians, no country can call itself free.

This involves the whole problem of scientific

and technical training from secondary

education to fundamental research..."

RenéMaheu, UNESCO Director General (1965)

\section{The ICTP TRIL Programme:}

Training and Research in

Italian Laboratories

\author{
G. Furlan, \\ The ICTP TRIL Programme, Trieste, Italy
}

$T$ he International Centre For Theoretical Physics (ICTP) in TriProgramme for Training and Research in Italian Laboratories (TRIL). The main motivation was the increasing demand from many developing countries scientists to have an advanced experimental counterpart to the theoretical research and lecture-based training offered at the Trieste Centre. ${ }^{1}$

A more farsighted view was to favour, through direct contacts and side-by-side research, the regular development of collaborations between the Italian scientific community and individuals, groups, and institutions in developing countries, enlarging substantially the line of action of the ICTP. The main objective remains to strengthen a permanent elite which, being aware of the needs of their own country and cognisant of the frontiers of science and technology, may properly influence the decision-makers' choices.

The specific purpose of the TRIL Programme is to offer scientists from developing countries who have participated in the ICTP scientific activities (conferences, workshops, schools), the opportunity of widening their experience by getting actively involved, in different branches of physical sciences, with the research work of laboratories at Italian universities and at public and private research centres. This includes academic studies as well as practical applications and industrial projects. In general, stays in the laboratory last several months (mostly one year and longer), but shorter visits are also envisaged.

The fields covered, which reflect current activities held at the ICTP, can be broadly classified as ${ }^{2}$ :

- Physics of Condensed Matter

- Physics and Energy

- Physics and Technology

- Earth and Environmental Sciences

- Physics of the Living State

- Miscellaneous (Instrumentation, Topics at the interface with other sciences i.e. Chemistry, Biology, Mathematics) 
Grants in a specific area are announced by a poster which contains a list of the Italian Laboratories agreeable to host scientists from developing countries together with a short presentation of the research activity carried out in each of them.

The selection is done jointly with the Italian laboratory indicated by the candidate as a priority in his application and is based mostly on scientific merit and on the matching of the candidate's expertise to the research lines pursued in the laboratory.

\section{Achievements}

The TRIL Programme represents no doubt one of the most successful and fruitful activities of the centre. Since 1983, in this framework 981 scientists (for a total of 1428 grants and 13,198 person-months) from developing countries have been offered many interesting opportunities to participate in side-by-side high level research, mostly experimental, working in active Italian teams with advanced equipment and experiencing an international atmosphere (see table 1).

\section{Table 1:}

$\begin{array}{lc}\text { Grants awarded } & 1428 \\ \text { Grants of less than } 3 \text { months } & 366 \\ \text { Grants of more than } 3 \text { months } & 1062 \\ \text { Fellows } & 981^{\star} \\ \text { Person-months } & 13198 \\ \text { Laboratories involved } & 339 \\ \text { Publications submitted } & 2773^{* *} \\ \text { Countries involved } & 73\end{array}$

* Several fellows were awarded more than one grant.

** Results obtained from the 1348 grants terminated.

It can also be interesting to have a geographical distribution of grants and fellows. This is presented in table 2 which shows the figures relevant to the ten countries which have till now better profited by TRIL.

$\checkmark$ Table 2:

\begin{tabular}{lccc}
\hline Country & Applications & Grants & Fellows \\
China & 1596 & 272 & 208 \\
India & 1288 & 271 & 185 \\
Argentina & 220 & 91 & 64 \\
Nigeria & 502 & 68 & 40 \\
Brazil & 88 & 41 & 37 \\
Cuba & 139 & 59 & 37 \\
Egypt & 360 & 40 & 26 \\
Turkey & 156 & 33 & 23 \\
Poland & 263 & 30 & 23 \\
Romania & 129 & 38 & 20 \\
\hline
\end{tabular}

More than 330 Italian laboratories have until now contributed to TRIL without any charge for the assistance offered to the fellow. Universities are the most numerous host institutions because of their historical ability of dealing with different problems and interesting solutions. On the other hand laboratories of public and private research institutions-CNR, ENEA, INFN ${ }^{3}$ and several others-often dispose of equipment which is better geared to a specific research and the presence of foreign visitors can be a valid solution to temporary personnel problems. The stay in Italy also represents a rewarding cultural and human experience, even more if the fellow is accompanied by his family members.

As a measure of the success one can mention the often significant contribution offered by the fellow to the research activity of the laboratory, the good standard of the reports published, the fact that frequently a TRIL fellowship, considered a guarantee of academic excellence, has been instrumental for the scientists to progress in his academic carrier (to the rank of Full Professor, Vice Chancellor, High Ministerial Official, even Minister). Another positive aspect is represented by the ever increasing interest and participation of the Italian scientific institutions which continue to
The TRIL Programme represents one of the most successful and fruitful activities of the centre. offer the possibility of a high level scientific collaboration and often contribute financially to the costs of the grant, sometimes through specific agreements (in 2002 the financial contribution from those sources constituted more than half of the TRIL budget!).

\section{Developments}

The visit of an individual scientist has in many cases constituted the seed for a more extended collaboration, which sometimes involves the institutions. One can quote the fruitful, almost regular collaboration between Italian Laboratories and corresponding institutions in India, China, Cuba, Argentina, Morocco, Nigeria. This side of the programme, i.e. the "follow-up" stage, represents one of the main objectives of the TRIL and needs continued attention and support. Many return visits have been supported but in order to make the "follow up" action more effective, the creation of a TRIL Associate Scheme was recently advocated. Paralleling the original ICTP-Associate Programme, the TRIL Associates are entitled to pay regular visits to the laboratory of their first stage, 3 visits in 5 years, $2-3$ months each time, to complete and update original research projects.

Twenty years later, the above description confirms that the TRIL Programme can be considered a very valuable component of the action of the ICTP (and of the Italian Government) to strengthen a scientific-technological elite in the developing world, in the broader framework of the relations between the North and the South. A natural development of TRIL is a series of "more," more topics to be included, more fellowships, more collaborations etc. But also more industrialized countries supporting our endeavour: partners from Europe are welcome any time! Will our physicists' community be willing to set up a TREL (E for Europe) Programme?

\section{Footnotes}

1 For a more complete description of the motivations, achievements, programmes of the Abdus Salam ICTP see "The Constant yet EverChanging Abdus Salam International Centre for Theoretical Physics", Juan G. Roederer, Physics Today, v. 54, n. 9, September 2001, pp. 31-36.

2 Purposely Experimental Particle Physics was not initially considered as a field of primary interest to developing countries.

3 CNR; National Research Council ENEA: National Body for Energy and Environment INFN: National Institute for Nuclear Physics 